\title{
A New Approach to Camera Image Indexing
}

\author{
Rastislav Lukac and Konstantinos N. Plataniotis \\ The Edward S. Rogers Sr. Dept. of Electrical and Computer Engineering, \\ University of Toronto, 10 King's College Road, Toronto, M5S 3G4, Canada \\ \{lukacr, kostas\}@dsp.utoronto.ca \\ http://www.dsp.utoronto.ca/ lukacr
}

\begin{abstract}
This paper presents a color filter array (CFA) image indexing approach. To enhance the functionality of single-sensor consumer electronics such as digital cameras, imaging-enabled mobile phones and wireless personal digital assistants (PDAs), the proposed solution embeds the metadata information to a CFA image using a common discrete cosine transform (DCT) based watermarking scheme. Depending on a consumer electronic device employed, the metadata information can be used to indicate ownership, capturing device identification numbers, time and location information. The metadata information can be extracted from the gray-scale, mosaic-like CFA image or the full-color, demosaicked image using PC software commonly available by camera manufacturers or with conventional public image database tools. Simulation studies reported in the paper indicate that the proposed CFA indexing approach does not affect the performance of the demosaicking methods which produce full-color images that are visually identical to those obtained by demosaicking of the non-indexed CFA data.
\end{abstract}

\section{Introduction}

Cost-effective imaging devices use a single image sensor, usually a charge-coupled device (CCD) or complementary metal oxide semiconductor (CMOS) sensor, to capture a visual scene [1]-5]. Due to the monochromatic nature of the sensor, a color filter array (CFA) is placed at the top of the sensor to capture the RedGreen-Blue (RGB) primary colors at the same time 3. Since each sensor cell has its own spectrally selective filter, the CFA sensor values constitute a mosaic-like gray-scale image (Fig 1]a) [4. The full-color RGB image (Fig 1 c) is obtained by estimating the two missing color components at each spatial location of the CFA image using a process called demosaicking [5]-9].

Single-sensor devices store a captured image either in CFA or demosaicked formats. To organize and retrieve the captured images in personal databases, and authenticate the visual material available to public, a single-sensor captured image should be naturally connected to digital databases using metadata [10, [1] embedded in the CFA domain. The metadata information can be extracted from either the CFA images or their demosaicked variants in both personal and public image databases using the processing routines to be built in PC software commonly available by camera manufacturers and public database programmers. 


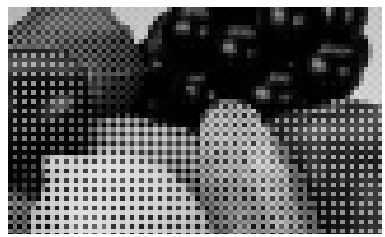

(a)

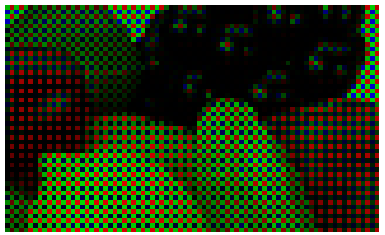

(b)

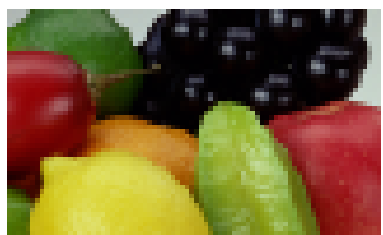

(c)

Fig. 1. Single-sensor imaging: (a) raw sensor data, (b) CFA data arranged as a color image, (c) reconstructed, full-color, demosaicked image

\section{Bayer CFA Based Single-Sensor Imaging}

Let us consider a single-sensor imaging pipeline equipped with a Bayer CFA (Fig 2) [12. The captured sensor data constitute a $K_{1} \times K_{2}$ gray-scale, mosaiclike, CFA image $z: Z^{2} \rightarrow Z($ Fig 1 $1 a)$ of integer samples $z_{(r, s)}$, with $r=$ $1,2, \ldots, K_{1}$ and $s=1,2, \ldots, K_{2}$ denoting the image rows and columns, respectively. This CFA image $z$ can be transformed to a $K_{1} \times K_{2}$ color (RGB) image $\mathbf{x}: Z^{2} \rightarrow Z^{3}\left(F i g[1 b)\right.$ of RGB vectors $\mathbf{x}_{(r, s)}=\left[x_{(r, s) 1}, x_{(r, s) 2}, x_{(r, s) 3}\right]$ with $x_{(r, s) k}$ indicating the $\mathrm{R}(k=1), \mathrm{G}(k=2)$ and $\mathrm{B}(k=3)$ component. Similarly to $z$, the vector field $\mathbf{x}$ have a mosaic-like structure due to the fact that the vector $\mathbf{x}_{(r, s)}$, such as $\mathbf{x}_{(r, s)}=\left[z_{(r, s)}, 0,0\right]$ for (odd $r$, even $\left.s\right), \mathbf{x}_{(r, s)}=\left[0,0, z_{(r, s)}\right]$ for $($ even $r$, odd $s)$, and $\mathbf{x}_{(r, s)}=\left[0, z_{(r, s)}, 0\right]$ for (odd $r$, odd $s$ ) and (even $r$, even $\left.s\right)$, contains the values which correspond to different spectral bands [4, [6].

The full-color image (Fig 1 c) is recovered from the CFA image using the socalled demosaicking process which calculates the missing color components from the adjacent CFA data 4, 9]. Depending on the device and the demosaicking solution employed, the quality of the demosaicked image as well as the computational complexity can vary significantly [4, 6]. Since the demosaicked images often suffer from zipper effects, reduced sharpness, and false coloration which result in various visual impairments, the postprocessing steps should be used to complete the demosaicking process [13, 14].

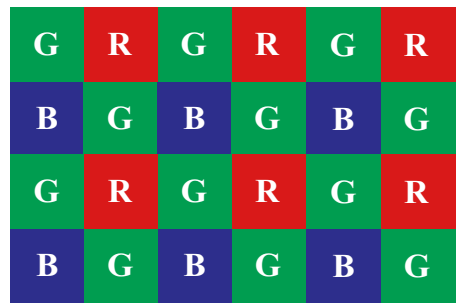

Fig. 2. Bayer CFA pattern with a GRGR phase in the first row 12 


\section{Proposed CFA Image Indexing Approach}

The demosaicking solutions are conventionally implemented in a digital camera which stores the demosaicked output. Alternatively, demosaicking is performed in a companion personal computer (PC) which interfaces with the digital camera that stores the images in the raw CFA format. Therefore, a single-sensor captured image should be naturally connected to digital databases using metadata embedded in the CFA domain [10. As it is shown in Fig [3, the metadata can vary in the type and amount of the information to be processed, for example: i) digital cameras can automatically use their identification number, ownership information and a time stamp, ii) imaging enabled phones can complete the camera's metadata information by adding location stamps, and iii) semantic content can be optionally added using the mobile phone's or pocket device's keyboard.

The solution in [10] processes the metadata information using image sharing concepts [15]-21 and encrypts the metadata information into two shares. In the sequence, the highest perceptual quality of the captured image is obtained by embedding the metadata share information at the least significant bits (LSB) of $\mathrm{R}$ and $\mathrm{B}$ CFA samples.

By embedding the metadata information in the frequency domain of the CFA image through digital watermarking (DW) concepts, the proposed here approach enhances efficiency of the CFA indexing approach and increases the robustness against signal processing operations. In addition, it twice reduces the amount of the embedded information compared to the information embedded through the secret sharing concept in 10. Note that the proposed solution can employ any DW scheme operating in the frequency domain. To demonstrate the concept, the conventional discrete cosine transform (DCT) based DW solution [22]-[24] is used in the sequence.

Following the dominance of $\mathrm{G}$ values $(50 \%)$ in the Bayer CFA which greatly contribute to the perceived sharpness of the demosaicked image [8], 9], our solution embeds the metadata information to the spatial locations corresponding to the $\mathrm{R}$ or B CFA components. It will be shown that by operating on the indexed CFA image, the subsequent demosaicking procedure produces a demosaicked image which is visually identical to the one reconstructed using the non-indexed, original CFA data.

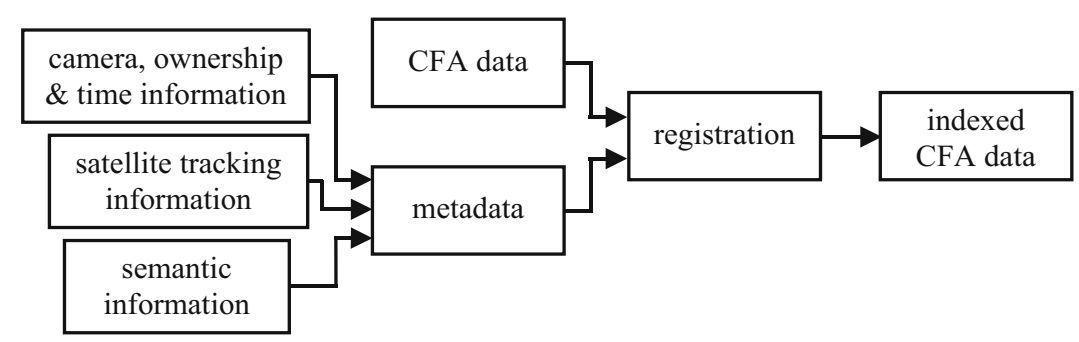

Fig. 3. A CFA image indexing concept 

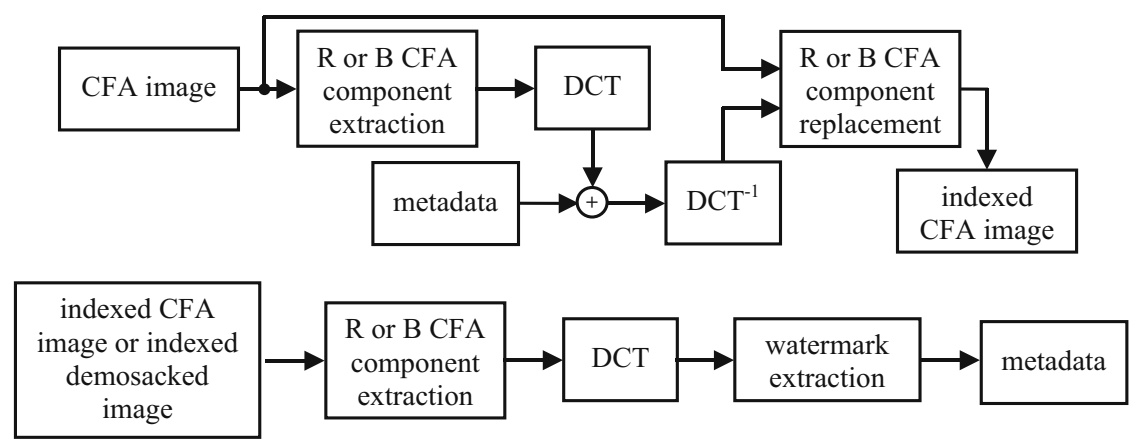

Fig. 4. Simplified block scheme representation of the proposed CFA image indexing solution: (top) metadata embedding, (bottom) metadata extraction

Using the conventional DCT based DW framework 22]-24, the metadata information is considered here as a binary image. As it is shown in Fig 4 the highest perceptual quality of the captured image is ensured by performing the indexing operations over the $\mathrm{R}$ or $\mathrm{B}$ components of the captured data. To demonstrate the concept, only the R CFA locations were considered in this paper to be affected by the indexing operations and thus, the proposed solution firsts extracts all the R CFA components. Following the conventional practice, the DCT is applied in blocks of $8 \times 8$ pixels. The metadata information is embedded in the DCT transform domain of the image constituted by the R CFA components. The metadata embedding process was controlled by global parameter $\alpha$ used to amplify or attenuate the watermark at each DCT coefficient. By tuning the value of $\alpha$, the tradeoff between the maximization of watermark energy (and robustness of the watermark) and imperceptibility of the changes introduced by watermarking is controlled. In this paper we used the value $\alpha=3$.

Based on the extracted metadata information, the indexed single-sensor captured images (both CFA images and demosaicked images) can be archived, uniquely organized, and retrieved in digital databases [10. The original CFA components, as described in Section 2, are not affected by the demosaicking procedure and thus, they are present in both CFA and demosaicked images. The database tools are used to extract the R CFA components in order to recover the original metadata information (Fig 4 ).

\section{Experimental Results}

To demonstrate the performance of the proposed method, a number of color and binary (metadata) test images have been used. The test color images, such as those shown in Figs 5 a $a$ - $d$ have been captured using three-sensor devices and normalized to 8-bit per channel RGB representation. The example of a binary metadata image is shown in Fig 5le. 


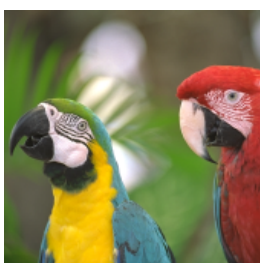

(a)

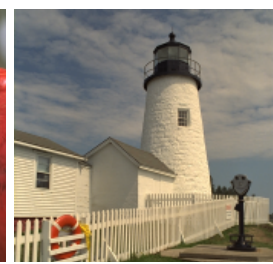

(b)

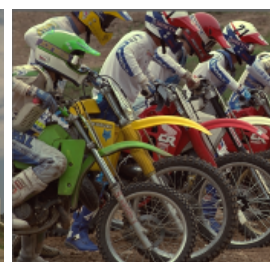

(c)

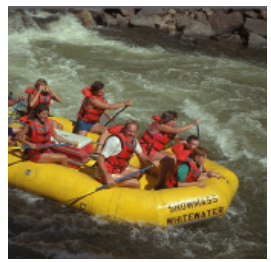

(d)

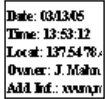

(e)

Fig. 5. Test images: (a-d) $512 \times 512$ color images Parrots, Lighthouse, Bikes and Rafting, (e) $64 \times 64$ binary metadata image

Table 1. Demosaicking performance using the BI scheme

\begin{tabular}{c|rrr|rrr}
\hline Input CFA image & \multicolumn{3}{|c|}{ non-indexed } & \multicolumn{3}{c}{ indexed } \\
\hline Image / Criterion & $M A E$ & $M S E$ & $N C D$ & $M A E$ & $M S E$ & $N C D$ \\
\hline Parrots & 2.087 & 29.5 & 0.0265 & 2.149 & 29.7 & 0.0268 \\
Lighthouse & 4.555 & 129.5 & 0.0579 & 4.612 & 129.7 & 0.0579 \\
Bikes & 6.060 & 155.5 & 0.1205 & 6.114 & 155.7 & 0.1208 \\
Rafting & 4.711 & 89.4 & 0.0750 & 4.746 & 89.6 & 0.0752 \\
\hline
\end{tabular}

Following the conventional practice, the mosaic versions of the original color images were created by discarding color information in a GRGR phased Bayer CFA filter shown in Fig 2. The indexed version of the CFA image was produced via the proposed CFA image indexing solution (Fig.4). The demosaicked and indexed demosaicked images were respectively obtained from non-indexed and indexed images by applying the bilinear demosaicking (BI) solution of [25] and the color-correlation demosaicking approach (CCA) of [1. Note that the BI scheme is commonly accepted as an industry standard whereas the CCA scheme is one of the most powerful demosaicking solutions.

The difference between the original images and the demosaicked images, as well as the original images and the indexed demosaicked images was evaluated using the mean absolute error (MAE), the mean square error (MSE) and the normalized color difference criterion (NCD). The interesting reader can find the definition of the above criteria in [1, 9]. Demosaicking results reported in $T a$ bles 1, 2 indicate that the demosaicked images obtained using both non-indexed and indexed CFA data are of similar quality. The comparison of the results also reveals the qualitative difference between the simple BI scheme and the sophisticated CCA solution. In both cases, demosaicking of the non-indexed data led to slightly better results.

Since the demosaicked images are intended for human inspection, visual comparisons are provided in Fig [6. As it can be seen, visual inspection does not reveals any difference by comparing the non-indexed BI demosaicked (Fig $\lcm{6} b$ ) 
(a)

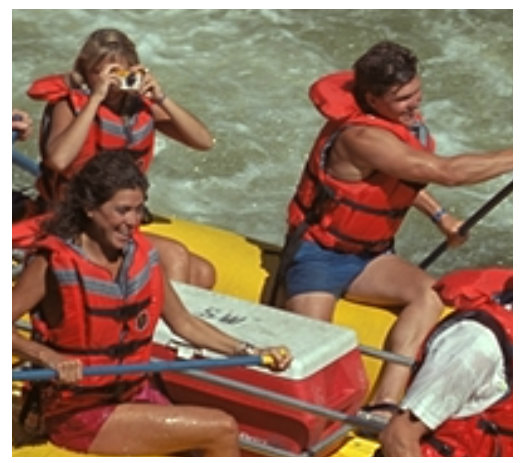

(b)
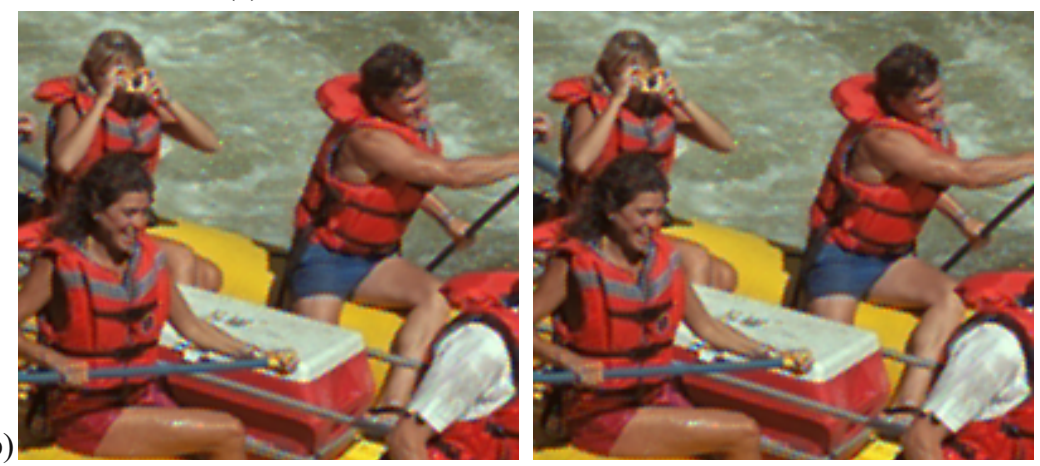

(c)

(d)
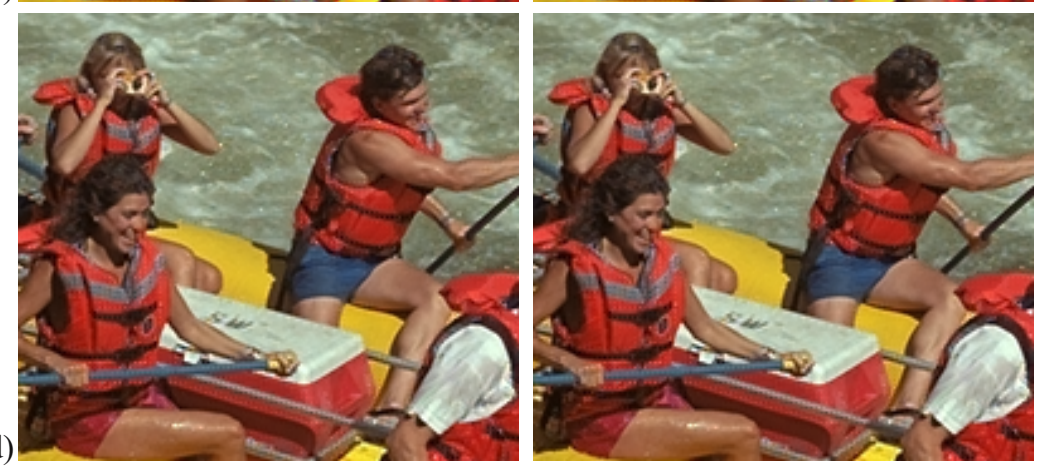

(e)

Fig. 6. Detailed parts of the obtained results: (a) original image, (b,c) BI demosaicking, (d,e) CCA demosaicking, (b,d) demosaicking using non-indexed CFA data, (c,e) demosaicking using indexed CFA data

and indexed BI demosaicked (Fig $[$ G ) images. Similarly, no difference between non-indexed and indexed demosaicked images can be observed by inspecting the images produced using the CCA demosaicking method (Figs, 6 d,e). It should be noted that the various visual impairments present in the BI demosaicked images are caused by the lack of spatial and spectral information during the BI demosaicking process. In the summary, it can be said that the proposed solution 
Table 2. Demosaicking performance using the CCA scheme

\begin{tabular}{c|rrr|rrr}
\hline Input CFA image & \multicolumn{3}{|c|}{ non-indexed } & \multicolumn{3}{c}{ indexed } \\
\hline Image / Criterion & $M A E$ & $M S E$ & $N C D$ & $M A E$ & $M S E$ & $N C D$ \\
\hline Parrots & 1.062 & 4.2 & 0.0151 & 1.123 & 4.4 & 0.0154 \\
Lighthouse & 1.455 & 9.3 & 0.0193 & 1.513 & 9.5 & 0.0195 \\
Bikes & 1.772 & 12.2 & 0.0428 & 1.829 & 12.3 & 0.0432 \\
Rafting & 1.725 & 14.1 & 0.0308 & 1.783 & 14.2 & 0.0311 \\
\hline
\end{tabular}

preserves the perceptual quality of both the CFA and demosaicked images. In addition, it does not introduce any side effect nor decrease the measured and/or visual quality of the captured image.

\section{Conclusion}

A new CFA image indexing approach was presented. Digital watermarking concepts were used to embed metadata into the single-sensor captured image in an imperceivable way. The extraction procedure recovers the original metadata from either indexed CFA or demosaicked image.

\section{References}

1. Lukac, R., Plataniotis, K.N., Hatzinakos, D., Aleksic, M.: A novel cost effective demosaicing approach. IEEE Transactions on Consumer Electronics 50 (2004) 256261

2. Lukac, R., Martin, K., Plataniotis, K.N.: Digital camera zooming based on unified CFA image processing steps. IEEE Transactions on Consumer Electronics 50 (2004) $15-24$

3. Adams, J., Parulski, K., Spaulding, K.: Color processing in digital cameras. IEEE Micro 18 (1998) 20-30

4. Lukac, R., Plataniotis, K.N.: Normalized color-ratio modelling for CFA interpolation. IEEE Transactions on Consumer Electronics 50 (2004) 737-745

5. Lu, W., Tang, Y.P.: Color filter array demosaicking: new method and performance measures. IEEE Transactions on Image Processing 12 (2003) 1194-1210

6. Lukac, R., Plataniotis, K.N.: Data-adaptive filters for demosaicking: a framework. IEEE Transactions on Consumer Electronics, 51 (2005)

7. Pei, S.C., Tam, I.K.: Effective color interpolation in CCD color filter arrays using signal correlation. IEEE Trans. Circuits and Systems for Video Technology 13 (2003) 503-513

8. Gunturk, B., Altunbasak, Y., Mersereau, R.: Color plane interpolation using alternating projections. IEEE Transactions on Image Processing 11 (2002) 997-1013

9. Lukac, R., Plataniotis, K.N., Hatzinakos, D.: Color image zooming on the Bayer pattern. IEEE Trans. on Circuit and Systems for Video Technology 15 (2005) 
10. Lukac, R., Plataniotis, K.N.: Digital image indexing using secret sharing schemes: a unified framework for single-sensor consumer electronics. IEEE Trans. Consumer Electronics, submitted (2004)

11. Toyama, K., Logan, R., Roseway, A., Anandan, P.: Geographic location tags on digital images. Proc. ACM International Conference on Multimedia in Berkeley, USA, (2003) 156-166.

12. Bayer, B.E.: Color imaging array. U.S. Patent 3971065 (1976)

13. Lukac, R., Martin, K., Plataniotis, K.N.: Demosaicked image postprocessing using local color ratios. IEEE Transactions on Circuit and Systems for Video Technology 14 (2004) 914-920

14. Lukac, R., Plataniotis, K.N.: A robust, cost-effective postprocessor for enhancing demosaicked camera images. Real-Time Imaging, Special Issue on Spectral Imaging II, $11(2005)$

15. Naor, M., Shamir, A.: Visual Cryptography. Lectore Notes in Computer Science 950 (1994) 1-12

16. Yang, C.N.: New visual secret sharing schemes using probabilistic method. Pattern Recognition Letters, 25 (2004) 481-494

17. Yang, C.N., Chen, T.S: Aspect ratio invariant visual secret sharing schemes with minimum pixel expansion. Pattern Recognition Letters, 26 (2005) 193-206

18. Ateniese, G., Blundo, C, de Santis, A., Stinson, D.G.: Visual cryptography for general access structures. Information and Computation 129 (1996) 86-106

19. Lukac, R., Plataniotis, K.N.: Bit-level based secret sharing for image encryption. Pattern Recognition 38 (2005) 767-772

20. Lukac, R., Plataniotis, K.N.: Colour image secret sharing. IEE Electronics Letters 40 (2004) 529-530

21. Lukac, R., Plataniotis, K.N.: Image representation based secret sharing. Communications of the CCISA, Special Issue on Visual Secret Sharing 11 (2005) 103-114

22. Hernandez, J.R., Amado, M., Perez-Gonzalez, F., DCT-domain watermarking techniques for still images: detector performance analysis and a new structure. IEEE Transactions on Image Processing 9 (2000) 55-68.

23. Kejariwal, A.: Watermarking. IEEE Potentials 22 (2003) 37-40

24. Barni, M., Bartolini, F., Piva, A.: Multichannel watermarking of color images. IEEE Transactions on Circuits and Systems for Video Technology 12 (2002) 142156

25. Sakamoto, T., Nakanishi, C., Hase, T.: Software pixel interpolation for digital still cameras suitable for a 32-bit MCU. IEEE Transactions on Consumer Electronics 44 (1998) 1342-1352 